\title{
Spontaneous Upbeat Nystagmus and Selective Anterior Semicircular Canal Hypofunction on Video Head Impulse Test: A New Variant of Canalith Jam?
}

\author{
Andrea Castellucci ${ }^{1}$, Cecilia Botti ${ }^{1,2}$, Salvatore Martellucci ${ }^{3}$, Pasquale Malara ${ }^{4}$, \\ Silvia Delmonte ${ }^{1}$, Francesca Lusetti ${ }^{1}$, and Angelo Ghidini ${ }^{1}$ \\ ${ }^{1}$ ENT Unit, Department of Surgery, Azienda USL-IRCCS di Reggio Emilia, Reggio Emilia, Italy \\ ${ }^{2} \mathrm{PhD}$ Program in Clinical and Experimental Medicine, University of Modena and Reggio Emilia, Modena, Italy \\ ${ }^{3}$ ENT Unit, Santa Maria Goretti Hospital, Azienda USL Latina, Latina, Italy \\ ${ }^{4}$ Audiology \& Vestibology Service, Centromedico, Bellinzona, Switzerland
}

\author{
Received May 7,2021 \\ Revised August 18, 2021 \\ Accepted September 4, 2021 \\ Address for correspondence \\ Andrea Castellucci, MD \\ ENT Unit, Department of Surgery, \\ Azienda USL-IRCCS di Reggio \\ Emilia, Viale Risorgimento 80, \\ 42123 Reggio Emilia, Italy \\ Tel +39-0522-296273 \\ Fax +39-0522-295839 \\ E-mail andrea.castellucci@ausl.re.it
}

\begin{abstract}
We describe a rare case of spontaneous upbeat nystagmus (UBN) attributable to a canalith jam involving the anterior semicircular canal (ASC) in a patient in whom comprehensive vestibular assessment was useful to identify the underlying pathomechanism. A 56-year-old woman with unsteadiness following repositioning procedures for left-sided benign paroxysmal positional vertigo (BPPV) presented with spontaneous UBN that showed slight right torsional components. A vestibular test battery detected isolated left ASC hypofunction on a video-head impulse test (Video-HIT). We postulated a persistent utriculopetal deflection of the left ASC cupula, which was attributable to entrapment of debris in a narrow canal tract, with consequent sustained inhibition of the ampullary afferents. Although spontaneous UBN receded after impulsive physical therapy, unsteadiness deteriorated into positional vertigo secondary to canalolithiasis involving the ipsilateral posterior canal. In our view, physical therapy possibly fragmented the canalith jam and released free-floating otoconia that eventually settled into the ipsilateral posterior canal. Video HIT revealed normalization of ASC hypofunction, and leftsided posterior canal canalolithiasis was successfully treated using appropriate repositioning procedures. We propose that a canalith jam involving the ASC should be considered in the differential diagnosis of spontaneous UBN, particularly in patients with a history of BPPV and isolated ASC hypofunction detected on video HIT. J Audiol Otol 2022;26(3):153-159
\end{abstract}

Keywords: Vertical nystagmus; Otoconia; Head impulse test; Benign paroxysmal positional vertigo; Semicircular canals.

\section{Introduction}

Spontaneous upbeat nystagmus (UBN), also known as primary position UBN, is a rare type of acquired nystagmus, usually attributed to disorders involving central oculomotor pathways [1]. Anecdotally, it has also been associated to inner ear pathologies [2], besides vestibular migraine in which peripheral and central mechanisms could be implicated [3]. Benign

This is an Open Access article distributed under the terms of the Creative Commons Attribution Non-Commercial License (https://creativecommons.org/licenses/by-nc/4.0/) which permits unrestricted non-commercial use, distribution, and reproduction in any medium, provided the original work is properly cited. paroxysmal positional vertigo (BPPV) is considered the most common labyrinthine disease where displaced otoconia usually settle the posterior semicircular canal (PSC) due to its undermost position. Diagnostic maneuvers should elicit paroxysmal UBN with ipsilesional torsional components (i.e. the top pole of the eyes beating toward the undermost ear) due to resulting ampullofugal excitatory flows within the affected PSC [1]. Conversely, in rare cases of anterior semicircular canal (ASC) involvement, positionings are expected to evoke downbeat nystagmus (DBN) due to an ampullofugal shift of freefloating particles exciting ASC afferents $[1,4]$. Nowadays, thanks to the accessibility of the video-head impulse test (Video-HIT) 
and vestibular-evoked myogenic potentials (VEMPs) for clinical purposes, a precise measurement of the activity of each inner ear sensor is easily provided.

\section{Case Report}

A 56-year-old woman presented at our attention with persistent unsteadiness following canalith repositioning maneuvers (CRM) for relapsing left-sided PSC-BPPV. Her clinical history was uneventful besides arterial hypertension and subclinical hypothyroidism. She denied recurrent headache and significant auditory symptoms. Video-Frenzel examination highlighted spontaneous UBN with subtle right-torsional components not affected by head position changes (Supplementary Video 1 in the online-only Data Supplement). Nystagmus was suppressed by visual fixation whereas it slightly increased its amplitude by skull vibrations and both Dix Hallpike positionings. Bedside testing excluded oculomotor abnormalities and neurological examination ruled out central disorders. Video-HIT performed using the ICS Impulse device (Otometrics, Natus Medical Inc, Taastrup, Denmark) (Fig. 1A) only detected a hypoactive left-sided ASC, whereas cervical and ocular-VEMPs for air-conducted sounds recorded using a 2-channel evoked potential acquisition system (Viking, Nicolet EDX, CareFusion, Heidelberg, Germany) were within normality ranges (Fig. 1B). Temporal bones high-resolution computed tomography excluded bony labyrinth defects (Fig. 2A), whereas brain-magnetic resonance imaging ruled out brainstem/cerebellar abnormalities. A canalith jam (CJ) involving the left ASC was postulated (Fig. 2B). The patient first received weekly sessions of uneventful CRM for ASC-BPPV. Then, she was instructed to perform Brandt-Daroff exercises at home. Some weeks later, unsteadiness receded whereas positional vertigo recurred. Whereas spontaneous UBN disappeared and left ASC hypofunction normalized (Fig. 3A), long-latency and low-amplitude positional UBN with subtle left-torsional components was detected on left Dix Hallpike position (Supplementary Video 2 in the onlineonly Data Supplement) consistent with left PSC-BPPV (Fig. 3B). She finally received successful Epley's CRM with symptoms receding.

The authors certify that they comply with the Principles of Ethical Publishing and declare that they acted in accordance with ethical standards laid down in the 1964 Declaration of Helsinki and its later amendments. Written informed consent was obtained from the patient for the publication of this case report, including all data and images. The local ethical committee of the Azienda USL-IRCCS di Reggio Emilia does not perform formal ethical assessment for case reports.

\section{Discussion}

$\mathrm{CJ}$ is a rare subtype of BPPV occurring when otoconia are assumed to aggregate, either spontaneously or following CRM, and occlude a narrow canal tract. It results in a persistent deflection (either utriculofugal or utriculopetal) of the cupula and in a blockage of endolymphatic flows between the clot and the cupula itself, generating spontaneous nystagmus [5-7]. While CJ involving the horizontal canal has demonstrated to result in spontaneous nystagmus mimicking an acute vestibular loss [6-8], it has already been reported how PSC-CJ could generate spontaneous DBN overlapping central oculomotor disorders [9-11]. In both cases, a reversible isolated hypofunction for the affected canal on video-HIT has demonstrated to represent a key data in the differential diagnosis [6,9-11]. In fact, according to the pathomechanism assumed for $\mathrm{CJ}$, as the cupula of the affected canal is supposed to be persistently bent either in excitatory or inhibitory direction, dynamic responses of the ampullary receptor would be markedly impaired, leading to a canal "pseudo-hyporeflexia" [5-7,9-11]. Once the otolith clot crumbles, otoconia are free to float again within the canal, without affecting cupular dynamics for the high-frequency domain, thus resulting in a restoration of video-HIT measurements [6,9-11]

To the best of our knowledge, a CJ involving the ASC has never been reported in the literature. In the present case, an ASC-CJ could likely be hypothesized as the underlying pathomechanism. In fact, besides the recent left-sided BPPV suggesting an ipsilesional otoconial dislodgment, a continuous ampullopetal inhibitory flow induced by a persistent positive pressure exerted by the otolith clot over the cupula of the left ASC could likely result in spontaneous UBN with subtle righttorsional components (Fig. 2B). It is reasonable to assume that the debris clot accidentally remained entrapped within a stenotic tract of the ampullary arm of the left ASC to induce a continuous inhibitory ampullopetal flow of endolymph facilitated by the gravity vector. On the contrary, it might be hypothesized that a continuous ampullofugal excitatory flow resulting in a spontaneous nystagmus aligning with the same ASC plane but with opposite direction (downbeating with left-torsional components) could have been expected if $\mathrm{CJ}$ had settled a stenotic tract of the non-ampullary arm of the left ASC. In fact, both mechanism (inhibitory and excitatory) have already been described in CJ [5-7,9-11]. The lack of clear right-torsional components in presenting UBN (Supplementary Video 1 in the online-only Data Supplement) is in accordance with ASC-BPPV physiology, as it has been observed how the activation of ASC afferents mainly results in vertical eye movements, probably due to the peculiar geometrical orientation of 

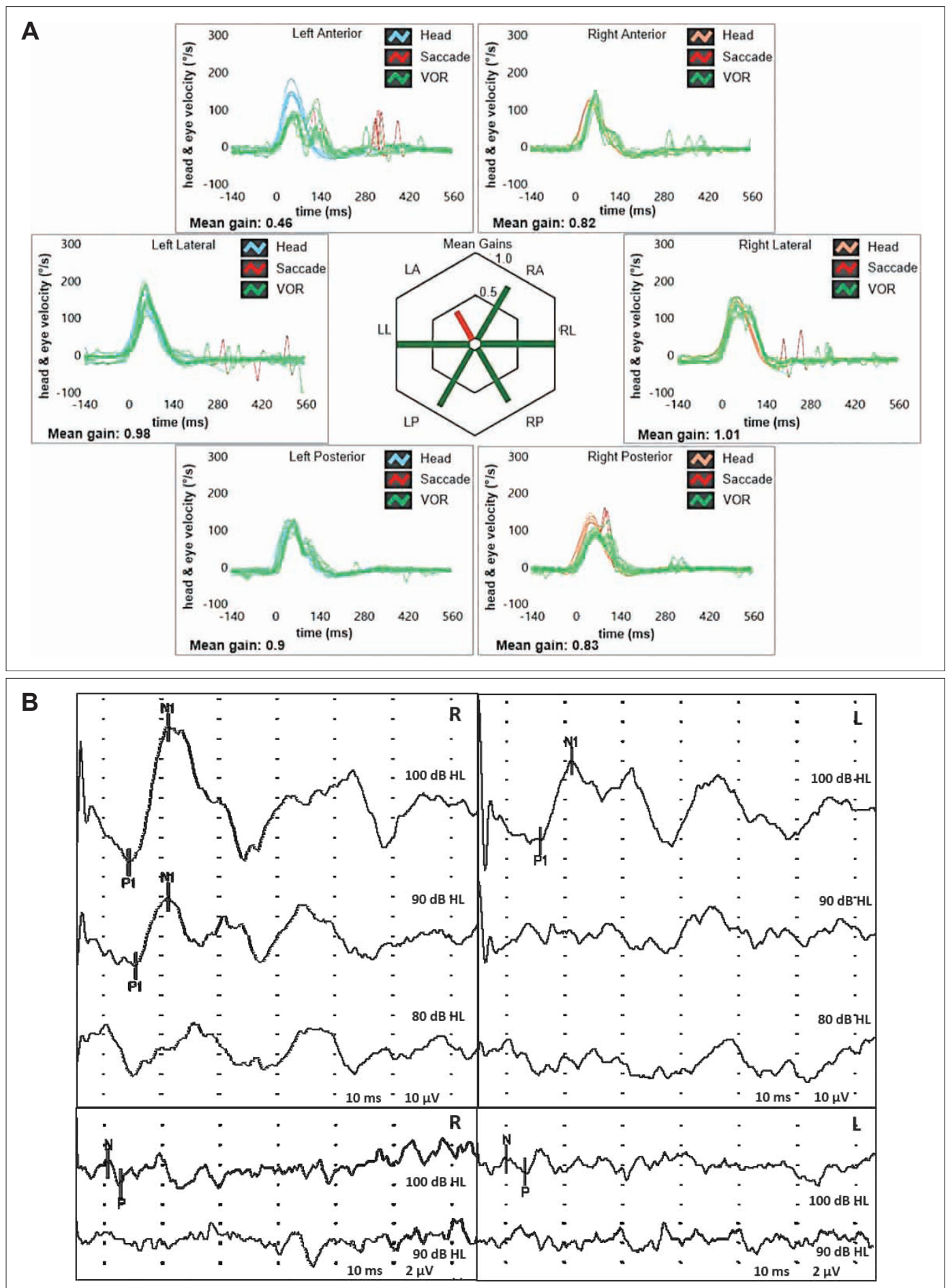

Fig. 1. Patient's vestibular assessment at presentation. A: Video-HIT performed using the ICS Impulse device (Otometrics, Natus Medical Inc, Taastrup, Denmark) assessing the VOR-gain (eye velocity/head velocity) for all six semicircular canals. Mean value of VORgain is reported for each canal. Gain values are considered normal if $>0.8$ for lateral canals and $>0.7$ for vertical canals. A selective reduction for the VOR-gain of the left ASC $(0.46)$ with both overt and covert saccades could be observed. B: Cervical (above) and ocularVEMPs (below) for air-conducted sounds recorded using a 2-channel evoked potential acquisition system (Viking, Nicolet EDX, CareFusion, Heidelberg, Germany). Potentials were recorded delivering tone bursts (frequency: $500 \mathrm{~Hz}$, duration: 8 ms, stimulation rate $5 \mathrm{~Hz}$ ) via headphones. VEMPs testing revealed slightly asymmetrical amplitudes for both cervical (R: $69.2 \mu \mathrm{V}$ and L: $41.4 \mu \mathrm{V}$ at $100 \mathrm{~dB}$ HL stimuli) and ocular-VEMPs (R: $2.2 \mu \mathrm{V}$ and L: $1.5 \mu \mathrm{V}$ for $100 \mathrm{~dB}$ HL sounds) with an asymmetry ratio within normality ranges for both measurements ( $25 \%$ and $19 \%$, respectively). ASC, anterior semicircular canal; HIT, head impulse test; L, left; LA, left anterior; LL, left lateral; LP, left posterior; R, right; RA, right anterior; RL, right lateral; RP, right posterior; VEMPs, vestibular-evoked myogenic potentials; VOR, vestibulo-ocular reflex. 

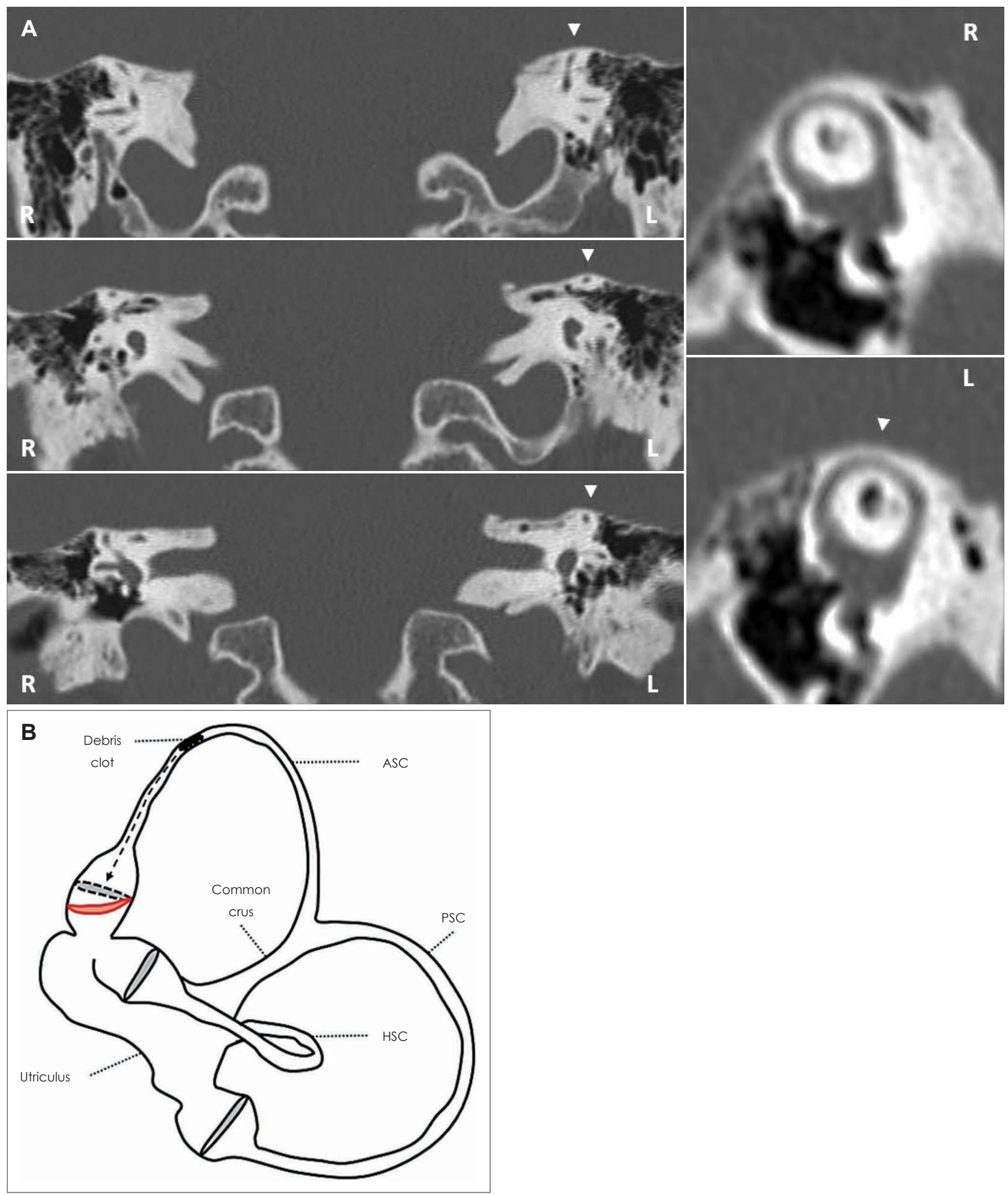

Fig. 2. Imaging and supposed pathomechanism for presenting symptoms and signs. A: Coronal images of temporal bone HRCT scan, with parasagittal reconstructions along the Pöschl planes, showing normal bony roof overlying the left ASC (white arrowhead). B: Schematic drawing of the left membranous labyrinth showing the assumed mechanism for presenting spontaneous UBN with subtle right torsional components and for selective left ASC hypofunction. A debris clot entrapped within the left ASC is supposed to generate a continuous positive pressure (black dashed arrow) on the cupula, inhibiting ampullary afferents and preventing dynamic responses for the canal by blocking endolymphatic flows. ASC-cupula is bent utriculopetally (red line) with respect to its original position (black dashed line). ASC, anterior semicircular canal; HRCT, high-resolution computed tomography; HSC, horizontal semicircular canal; L, left; PSC, posterior semicircular canal; R, right; UBN, upbeat nystagmus. 

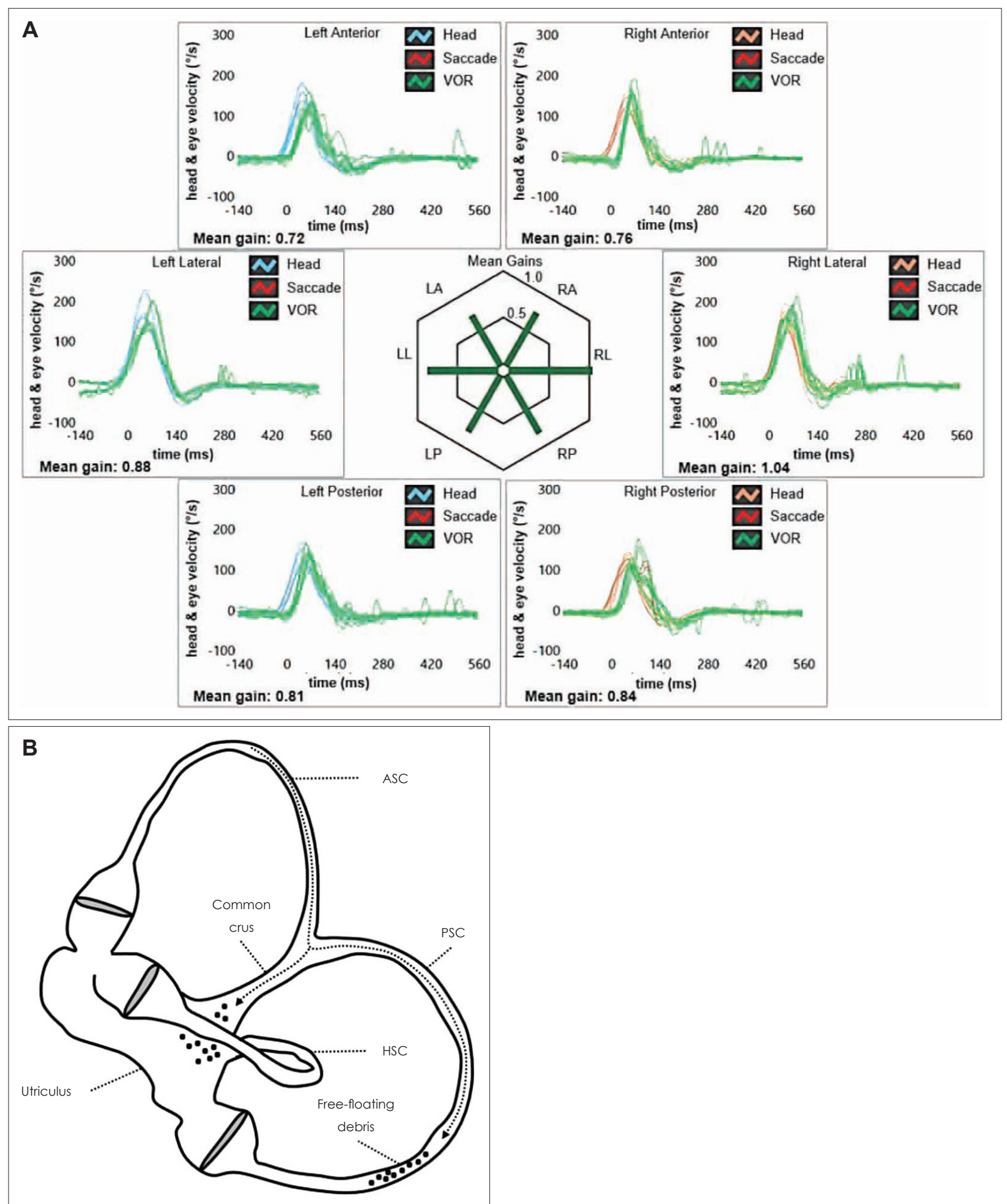

Fig. 3. Vestibular assessment and assumed pathomechanism for symptoms and signs following physical treatment. A: Video-HIT following physical treatment, confirming restitution of VOR-gain values for the left ASC (0.72). B: After impulsive physical treatment, the clot likely crumbled and part of otoconia eventually fell within the utriculus whereas a residual amount of free-floating debris settled the left PSC, crossing the common crus (black dotted arrow). ASC, anterior semicircular canal; HIT, head impulse test; HSC, horizontal semicircular canal; LA, left anterior; LL, left lateral; LP, left posterior; PSC, posterior semicircular canal; RA, right anterior; RL, right lateral; RP, right posterior; VOR, vestibulo-ocular reflex. 
the ASC and to an upwards bias in vertical slow phase eye velocity [4]. The unresponsiveness to standard CRM supports the assumption of an acquired/structural ASC stenosis preventing a complete release from otoconia. The final conversion in ipsilateral PSC-BPPV with Brandt-Daroff exercises suggests that an impulsive physical therapy was needed to disrupt the clot, allowing debris to overcome the canal stenosis and settle the PSC through the common crus (Fig. 3B). The same pathomechanism could also explain the underlying left ASC hypofunction, normalizing after physical treatment. On the other hand, neuroanatomical networks underlying vestibulo-olcular reflex (VOR) responses coming from the PSC may likely explain the lack of significant left-torsional components in transient positional UBN elicited in left-sided Dix Hallpike position due to the final left PSC-BPPV (Supplementary Video 2 in the online-only Data Supplement). In fact, according to anatomical and physiological data, PSC excitation results in activation of ipsilateral superior oblique and contralateral inferior rectus muscles, resulting in a disconjugate nystagmus with stronger torsional/horizontal components in ipsilateral eye movements and more vertical activity in the contralesional eye [1]. Since we adopted a head-mounted camera with right-eye monocular recording as video-Frenzel, positional nystagmus due to left PSC-BPPV resulted in stronger UBN than left-torsional components. Additionally, longlatency and low-amplitude eye movements evoked in left Dix Hallpike position could likely be explained hypothesizing that a portion of debris eventually fell within the utriculus after Brandt-Daroff exercises, whereas a residual smaller amount of particles crossed the common crus and settled the ampullary arm of the left PSC (Fig. 3B). In fact, according to a mathematical tridimensional model of the labyrinth, the latency of positional nystagmus in case of canalolithiasis would be inversely related to the size of floating particles, while the magnitude of eye movements would be directly related to the number of displaced otoconia [12]. Applying these notions in clinical practice, we could assume that few and small dislodged otoconia are expected to result in positional nystagmus with longer latency and small intensity, as in the case herein reported.

Finally, although ASC-BPPV has been related to positional vertigo rather than persistent unsteadiness as in the present case, it could be assumed that the presenting findings herein described could be attributed to left ASC-cupulolithiasis, where otoconia are thought to adhere to the cupula overloading it. Nevertheless, both ASC-canalolithiasis and cupulolithiasis should only result in positional DBN (either lasting less than 1 minute or persisting for more than 1 minute, respectively) after both Dix Hallpike and straight head hanging positionings, sometimes reversing when returning upright $[1,4]$. On the contrary, our patient presented with spontaneous UBN with slight right-torsional components enhanced by positioning tests, not reversing with head position changes. These findings could be explained neither by ASC-cupulolithiasis nor by ASC-canalolithiasis. The only possible mechanisms involving dislodged otoconia include either a continuous inhibitory endolymphatic flow bending the cupula of the left ASC towards the utricle due to left ASC-CJ or a continuous excitatory utriculofugal deflection of the right PSC cupula due to right PSC-CJ. Since the patient was previously treated for refractory left-sided PSC-BPPV and due to left ASC VORgain reduction consistent with $\mathrm{CJ}$ pathomechanism, it is reasonable to assume the first picture as the most likely hypothesis.

Although a selective ASC hypofunction on video-HIT has already been described in other peripheral pathologies including endolymphatic hydrops [13] and superior canal dehiscence [14], both conditions were excluded by clinical-instrumental data and imaging, respectively. Moreover, as central vestibular disorders have hardly demonstrated to result in a selective ASC hypoactivity [15], no other labyrinthine pathologies could likely result in spontaneous UBN with such a reversible lesion pattern.

In this report, we described how the use of a comprehensive vestibular assessment enabled to recognize a unique labyrinthine disorder as the underlying pathomechanism. Besides distinguishing from central disorders, a selective ASC hypofunction on video-HIT allowed to hypothesize an ASC-CJ and to treat the patient accordingly, providing further insights to the pathophysiology of spontaneous UBN of peripheral origin. Unfortunately, vestibular examination was conducted only with Video-Frenzel goggles, without a video-nystamographic acquisition system. Therefore, we could not provide nystagmographic data for spontaneous nystagmus (such as slow phase velocity) and for modulation of nystagmus amplitude according to positional changes and skull vibrations. Even though further investigations are needed before any conclusion can be reached on the clinical value of video-HIT in case of spontaneous nystagmus along the vertical plane, we can suggest to consider CJ involving the ASC in the differential diagnosis of spontaneous UBN, in particular if it is associated with previous BPPV and isolated ASC hypofunction on video-HIT.

\section{Supplementary Video}

Video 1. Presenting video-Frenzel examination showing spontaneous upbeat nystagmus with subtle torsional components beating towards the right side (upper eye poles), slightly enhanced by mastoid vibrations and by both Dix Hallpike positionings. 
Video 2. Post-treatment video-Frenzel examination showing total receding of spontaneous upbeating nystagmus. Left Dix Hallpike positioning test evoked paroxysmal upbeat nystagmus with left-torsional components consistent with BPPV involving the left posterior semicircular canal. A therapeutic Epley's repositioning maneuver is performed.

\section{Supplementary Materials}

The online-only Data Supplement is available with this article at https://doi.org/10.7874/jao.2021.00297.

\section{Acknowledgments}

None.

\section{Conflicts of interest}

The authors have no financial conflicts of interest.

\section{Author Contributions}

Conceptualization: Andrea Castellucci, Salvatore Martellucci, Pasquale Malara. Data curation: Andrea Castellucci, Cecilia Botti, Silvia Delmonte, Francesca Lusetti. Investigation: Andrea Castellucci, Cecilia Botti, Silvia Delmonte, Francesca Lusetti. Supervision: Angelo Ghidini. Writing — original draft: Andrea Castellucci. Writing-review \& editing: all authors. Approval of final manuscript: all authors.

\section{ORCID iDs}

Andrea Castellucci

Cecilia Botti

Salvatore Martellucci

Pasquale Malara

Silvia Delmonte

Francesca Lusetti

Angelo Ghidini https://orcid.org/0000-0003-2874-5672 https://orcid.org/0000-0002-0136-141X https://orcid.org/0000-0001-5233-2478 https://orcid.org/0000-0002-7475-9058 https://orcid.org/0000-0001-9522-1698 https://orcid.org/0000-0003-0153-9641 https://orcid.org/0000-0002-4117-1901

\section{REFERENCES}

1) Leigh RJ, Zee DS. The neurology of eye movements. 5th ed. New York: Oxford University Press;2015.

2) Reynard P, Karkas A, Gavid M, Lelonge Y, Bertholon P. Delayed endolymphatic hydrops. Special emphasis on nystagmus associated with episodes and contribution of chemical labyrinthectomy. Eur Ann Otorhinolaryngol Head Neck Dis 2018;135:321-26.

3) Young AS, Lechner C, Bradshaw AP, MacDougall HG, Black DA,
Halmagyi GM, et al. Capturing acute vertigo: a vestibular event monitor. Neurology 2019;92:e2743-53.

4) Bertholon P, Bronstein AM, Davies RA, Rudge P, Thilo KV. Positional down beating nystagmus in 50 patients: cerebellar disorders and possible anterior semicircular canalithiasis. J Neurol Neurosurg Psychiatry 2002;72:366-72.

5) Ko KM, Song MH, Kim JH, Shim DB. Persistent spontaneous nystagmus following a canalith repositioning procedure in horizontal semicircular canal benign paroxysmal positional vertigo. JAMA Otolaryngol Head Neck Surg 2014;140:250-2.

6) Castellucci A, Malara P, Brandolini C, Del Vecchio V, Giordano D, Ghidini A, et al. Isolated horizontal canal hypofunction differentiating a canalith jam from an acute peripheral vestibular loss. Am J Otolaryngol 2019;40:319-22.

7) Schubert MC, Helminski J, Zee DS, Cristiano E, Giannone A, Tortoriello G, et al. Horizontal semicircular canal jam: two new cases and possible mechanisms. Laryngoscope Investig Otolaryngol 2020; 5:163-7.

8) Martellucci S, Castellucci A, Malara P, Pagliuca G, Clemenzi V, Stolfa A, et al. Spontaneous jamming of horizontal semicircular canal combined with canalolithiasis of contralateral posterior semicircular canal. J Audiol Otol 2021 Feb 15 [Epub] https://doi.org/10.7874/ jao.2020.00507.

9) Castellucci A, Malara P, Martellucci S, Botti C, Delmonte S, Quaglieri S, et al. Feasibility of using the video-head impulse test to detect the involved canal in benign paroxysmal positional vertigo presenting with positional downbeat nystagmus. Front Neurol 2020; 11:578588.

10) Castellucci A, Malara P, Ghidini A. Spontaneous downbeat nystagmus in posterior semicircular canal benign paroxysmal positional vertigo: a canalith jam? Neurol Sci 2021;42:313-5.

11) Castellucci A, Malara P, Martellucci S, Delmonte S, Ghidini A. Fluctuating posterior canal function in benign paroxysmal positional vertigo depending on how and where otoconia are disposed. Otol Neurotol 2021;42:e193-8.

12) Rajguru SM, Ifediba MA, Rabbitt RD. Three-dimensional biomechanical model of benign paroxysmal positional vertigo. Ann Biomed Eng 2004;32:831-46.

13) Martinez-Lopez M, Manrique-Huarte R, Perez-Fernandez N. A puzzle of vestibular physiology in a Meniere's disease acute attack. Case Rep Otolaryngol 2015;2015:460757.

14) Castellucci A, Piras G, Del Vecchio V, Crocetta FM, Maiolo V, Ferri GG, et al. The effect of superior canal dehiscence size and location on audiometric measurements, vestibular-evoked myogenic potentials and video-head impulse testing. Eur Arch Otorhinolaryngol 2021;278:997-1015.

15) Choi JY, Kim HJ, Kim JS. Recent advances in head impulse test findings in central vestibular disorders. Neurology 2018;90:602-12. 